\title{
Quantitation of Varicella-Zoster Virus DNA in Patients with Ramsay Hunt Syndrome and Zoster Sine Herpete
}

\author{
YASUSHI FURUTA, ${ }^{1 *}$ FUMIO OHTANI,${ }^{1}$ HIROFUMI SAWA, ${ }^{2}$ \\ SATOSHI FUKUDA, ${ }^{1}$ AND YUKIO INUYAMA ${ }^{1}$ \\ Department of Otolaryngology ${ }^{1}$ and Laboratory of Molecular \& Cellular Pathology, CREST, JST, ${ }^{2}$ \\ Hokkaido University School of Medicine, Sapporo, Japan
}

Received 13 February 2001/Returned for modification 7 April 2001/Accepted 11 June 2001

\begin{abstract}
Varicella-zoster virus (VZV) reactivation causes facial nerve palsy in Ramsay Hunt syndrome (RHS) and zoster sine herpete (ZSH) with and without zoster rash, respectively. In the present study, we analyzed the VZV DNA copy number in saliva samples from 25 patients with RHS and 31 patients with ZSH using a TaqMan PCR assay to determine differences in the viral load between the two diseases. VZV copy number in saliva peaked near the day of the appearance of zoster in patients with RHS. Consequently, VZV DNA was less frequently detected in patients with RHS who exhibited facial palsy several days after the appearance of zoster. These findings suggest that the VZV load in saliva samples reflects the kinetics of viral reactivation in patients with RHS. In addition, VZV DNA was equally detected in saliva from patients with RHS and ZSH, and there was no significant difference in the highest viral copy number between patients with RHS and those with ZSH. The VZV load does not appear to reflect a major difference between RHS and ZSH.
\end{abstract}

Ramsay Hunt syndrome (RHS) is a varicella-zoster virus (VZV)-associated neurological disease, characterized by zoster around the ears or in the oropharynx and by acute peripheral facial palsy. In addition, RHS is frequently complicated by a disorder of the eighth cranial nerve. Facial palsy and zoster do not always appear simultaneously, and some patients with RHS exhibit facial palsy several days before or after the onset of zoster. VZV also causes acute peripheral facial palsy with the absence of skin lesions; such cases are termed zoster sine herpete $(\mathrm{ZSH})$ and are usually diagnosed using serological assays (11) or PCR $(3,12,13)$. Although VZV reactivation causes facial nerve disorder in both RHS and ZSH, no study has analyzed the difference in virological background between these two diseases. One hypothesis to explain the presence or absence of mucocutaneous lesions (zoster) is that the VZV load is higher in RHS than in ZSH.

Only a few studies have investigated the VZV load in patients with varicella and zoster. Studies using a TaqMan-based PCR assay have reported that the viral copy number of peripheral blood mononuclear cells (PBMCs) in patients with varicella was higher than that in patients with zoster $(6,10)$. However, in these studies, VZV DNA was detected in PBMCs from only $20 \%$ of patients with zoster. In addition, it has been demonstrated that VZV DNA was not detected in PBMCs from patients with ZSH (3). Judging from these reports, an analysis of PBMCs does not provide a useful measure of the VZV load in facial palsy patients.

Since it has been reported that VZV DNA is detectable in saliva samples from 58 to $59 \%$ of patients with RHS and ZSH (4), we employed a TaqMan PCR assay of DNAs from saliva samples for quantitation of VZV DNA and initially analyzed

* Corresponding author. Department of Otolaryngology, Hokkaido University School of Medicine, Kita 15, Nishi 7, Kita-Ku, Sapporo 060-8638, Japan. Phone: 81-11-707-3387. Fax: 81-11-717-7566. E-mail: yfuruta@med.hokudai.ac.jp. whether the viral copy number in saliva reflects the kinetics of VZV reactivation. We then compared the viral load between RHS and ZSH.

\section{MATERIALS AND METHODS}

Patient samples. Twenty-five patients with RHS and 31 patients with ZSH were examined. These patients visited our hospital within 7 days after the onset of the diseases. Acyclovir and prednisolone were administered to 21 of 25 patients with RHS and 17 of 31 patients with ZSH, according to a previously described protocol (5), while the remaining patients were treated with prednisolone. Saliva (1 to $2 \mathrm{ml}$ ) was collected from patients at every visit and stored at $-80^{\circ} \mathrm{C}$. Paired sera were taken from patients at their initial visit and 2 to 3 weeks later (convalescent phase). Anti-VZV antibody values were measured using enzyme-linked immunosorbent assay kits (Enzygnost Anti-VZV/IgG and IgM; Behringwerke, Marburg, Germany) and an automatic enzyme-linked immunosorbent assay processor (Processor III; Behringwerke). Either significant changes (greater than two fold) in immunoglobulin $\mathrm{G}(\mathrm{IgG})$ antibody values or the presence of $\operatorname{IgM}$ antibody was considered to indicate recent VZV infection, according to the manufacturer's recommendations. The diagnosis of $\mathrm{ZSH}$ was determined by PCR and/or serological assays as described previously (4). RHS was diagnosed by the clinical manifestation, including typical zoster lesions around the ear or in the oral epithelium in addition to acute peripheral facial palsy. VZV reactivation in patients with RHS was confirmed either by serological assays or by PCR. Informed consent was obtained from all patients.

TaqMan PCR. Saliva samples from patients were centrifuged at 2,200 $\times g$ for $5 \mathrm{~min}$, and DNA was extracted from $50 \mu \mathrm{l}$ of the supernatant with a DNA extraction kit (SepaGene; Sanko Junyaku Co., Tokyo, Japan). Detection of VZV DNA was performed by a TaqMan PCR method using a set of primers and a fluorogenic probe for the major DNA binding protein of VZV (1). The upstream and downstream primer sequences were 5'-GTGCTGTTGAGACGACCGG-3' and 5'-GGCTTCCTTAAACAATGCCG-3', respectively. The sequence of the fluorogenic probe was 5'-FAM-CTGAGATATGCACCCGCCTTGGATTA GA-TAMRA-3', which was labeled with 6-carboxy-fluorescein (FAM) reporter dye and 6-carboxy-tetramethyl-rhodamine (TAMRA) quencher dye. PCR amplification was performed using the TaqMan PCR kit (PE Applied Biosystems, Foster City, Calif.). Each $25-\mu$ l reaction mixture consisted of $5 \mu$ l of $10 \times$

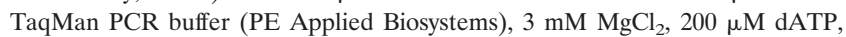
dGTP, and dCTP, $400 \mu \mathrm{M}$ dUTP, a $400 \mathrm{nM}$ concentration of each primer, 150 $\mathrm{nM}$ of the fluorogenic probe, $0.75 \mathrm{U}$ of AmpliTaq Gold, $0.01 \mathrm{U}$ of AmpErase uracil $N$-glycosylase (UNG), and template DNA. PCR mixtures were incubated for $2 \mathrm{~min}$ at $50^{\circ} \mathrm{C}$ for the reaction of AmpErase UNG, followed by activation of AmpliTaq Gold for $10 \mathrm{~min}$ at $95^{\circ} \mathrm{C}$. Reagents were cycled 45 times on a DNA thermal cycler (GeneAmp 5700 sequence detector; PE Applied Biosystems), 
TABLE 1. Detection of VZV DNA in patients with RHS and ZSH

\begin{tabular}{|c|c|c|c|c|}
\hline \multirow{2}{*}{ Disease $(n)$} & \multirow{2}{*}{ Appearance of zoster $(n)$} & \multirow{2}{*}{ No. (\%) of PCR-positive cases } & \multicolumn{2}{|c|}{$\begin{array}{l}\text { No. of PCR-positive cases with pattern of } \\
\text { VZV load }\end{array}$} \\
\hline & & & Gradual decrease & Peak after onset \\
\hline \multirow[t]{4}{*}{ RHS (25) } & Before onset of facial palsy (7) & $1(14)$ & 1 & 0 \\
\hline & Simultaneous (12) & $8(67)$ & 6 & 2 \\
\hline & After onset of facial palsy (6) & $4(67)$ & 0 & 4 \\
\hline & Total & $13(52)$ & 7 & 6 \\
\hline ZSH (31) & None & $17(55)$ & 10 & 7 \\
\hline
\end{tabular}

each cycle consisting of denaturation at $95^{\circ} \mathrm{C}$ for $15 \mathrm{sc}$, annealing, and extension at $62^{\circ} \mathrm{C}$ for $1 \mathrm{~min}$.

Quantitation of VZV DNA. The quantitation of VZV DNA in saliva samples was performed using a fluorescence detector (GeneAmp 5700 sequence detector). A threshold cycle value for each sample was determined by the cycle number at which the fluorescence exceeded a threshold limit (20 times the standard deviation of the baseline). The EcoRI B restriction fragment of VZV strain H-S1 (a gift from R. Hondo) was used as a standard positive control in each reaction. Saliva samples obtained from VZV-seronegative subjects were used as negative controls. The sensitivity of the TaqMan PCR assay for detection of VZV DNA was assessed by using a dilution series of the EcoRI B restriction fragment, mixed with DNA extracted from the saliva samples of a VZV-seronegative subject. The DNAs of herpes simplex virus (HSV) type 1, HSV type 2, human cytomegalovirus, Epstein-Barr virus, and human herpesvirus 6 were applied to the assay to confirm the specificity of the primer sets and fluorogenic probe.

A standard curve was generated by the threshold cycle values obtained from serial 10 -fold dilutions of the positive control containing $10^{1}$ to $10^{5}$ copies of the target gene. After two independent PCR assays for each sample, the average VZV copy number in $50 \mu$ l of saliva was calculated by comparison with the standard curve. A sample containing more than $10^{5}$ copies of VZV DNA was diluted 10 - or 100 -fold and subjected to the assay again.

Statistical analysis. Statistical analysis was performed using StatView version 4.5 software (Abacus Concepts Inc., Berkeley, Calif.). The chi-square test and Mann-Whitney U test were used to calculate the significance of the correlation among the variables. Differences with a $P$ value less than 0.05 were considered significant.

\section{RESULTS}

Sensitivity and specificity of the assay. The TaqMan PCR assay identified four of four samples containing 10 copies of the VZV DNA standard, while none of four samples containing 5 copies were positive by the assay. Based on these results, the minimum level of VZV DNA detectable by the TaqMan PCR was 10 copies. No cross-reactivity of the primer sets and fluorogenic probe was observed when the DNAs of HSV type 1, HSV type 2, human cytomegalovirus, Epstein-Barr virus, and human herpesvirus 6 were applied to this assay.

Quantitation of VZV DNA. In every assay, the standard curve showed linearity from 10 to $10^{5}$ copies of VZV DNA with correlation coefficients of $>0.96$. In a control study, the amount of total DNA extracted from $50 \mu \mathrm{l}$ of saliva was measured in 10 samples obtained from 10 control patients. The amounts of DNA ranged from 32 to $114 \mathrm{ng}$, probably due to contamination of epithelial cells. In order to evaluate the reliability of the quantitative PCR, a series of the VZV DNA standard $\left(10^{2}, 10^{3}\right.$, and $10^{4}$ copies $)$ was amplified together with different amounts (from 30 to $240 \mathrm{ng}$ ) of genomic DNA extracted from PBMCs of a VZV-seronegative subject in five repetitions. The amplification efficiency of VZV DNA was not altered in the presence of different amounts of genomic DNA (data not shown).

Detection of VZV DNA in saliva samples. VZV DNA was detected in saliva from 13 of $25(52 \%)$ patients with RHS and from 17 of $31(55 \%)$ patients with ZSH (Table 1$)$. The difference in the frequency was not significant $(P>0.05$, chi-square test).

Kinetics of the VZV load in RHS. We analyzed the correlation between the changes in the VZV copy number and the day of appearance of zoster in patients with RHS (Table 1). Seven patients exhibited facial palsy 2 or more days after the rash appeared; VZV DNA was detected in only one of the seven patients (14\%). Zoster and facial palsy developed simultaneously in 12 patients; VZV DNA was detected in 8 of the 12 patients $(67 \%)$. The VZV copy number gradually decreased after the first visit in six of the eight PCR-positive patients (Fig. 1A), while an increase in the viral copy number after the first visit was observed in the remaining two patients. In six patients, a rash developed 2 or more days after the onset of palsy, and the initial diagnosis for these patients was Bell's palsy. VZV DNA was detected in four of these six patients $(67 \%)$, and the viral copy number increased after the onset of palsy and peaked near the day of the appearance of zoster in all four patients (Fig. 1B). VZV DNA was detected in 11 of 21 patients with RHS who received acyclovir; the viral copy number showed a decrease in all except 1 patient within 2 days after initiation of the antiviral treatment (Fig. 1A and B).

Four patients had zoster in the oropharyngeal epithelium, and VZV DNA was detected in saliva samples from all four patients, while VZV DNA was detected in 9 of 21 patients who had zoster around the ear. The viral copy number in patients who had zoster in the oropharyngeal epithelium was significantly higher than that in patients who had zoster around the ear $(P=0.0019$, Mann-Whitney U test) (Fig. 2).

Kinetics of the VZV load in ZSH. In 10 of the 17 PCRpositive patients with $\mathrm{ZSH}$, the viral copy number gradually decreased after the first visit (Fig. 1C), while an increase in the viral DNA was observed in the remaining 7 patients (Table 1; Fig. 1D). Acyclovir was administered to 10 PCR-positive and 7 PCR-negative patients with ZSH. The viral copy number showed a decrease in 8 PCR-positive patients within 2 days after initiation of the antiviral treatment, as shown in Fig. 1C.

Difference in VZV copy number between RHS and ZSH. We then compared the VZV load in saliva from patients with RHS and from those with ZSH. Because the amount of VZV DNA in saliva varied on each sampling day, the highest copy number 
A
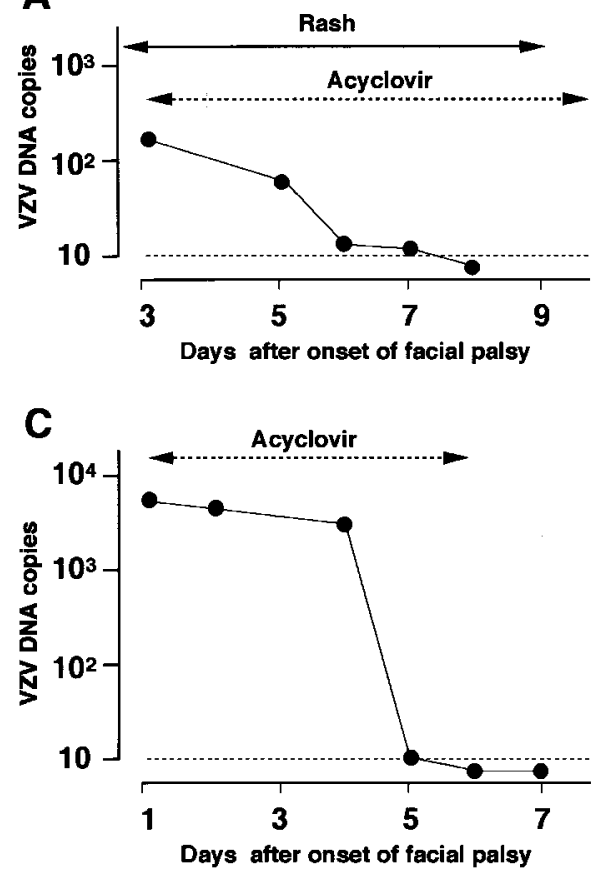

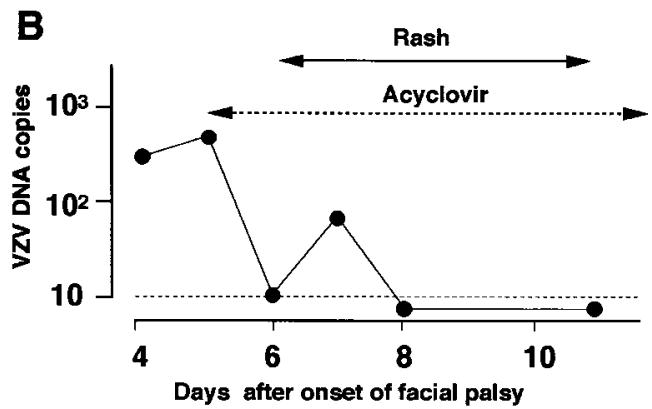

D

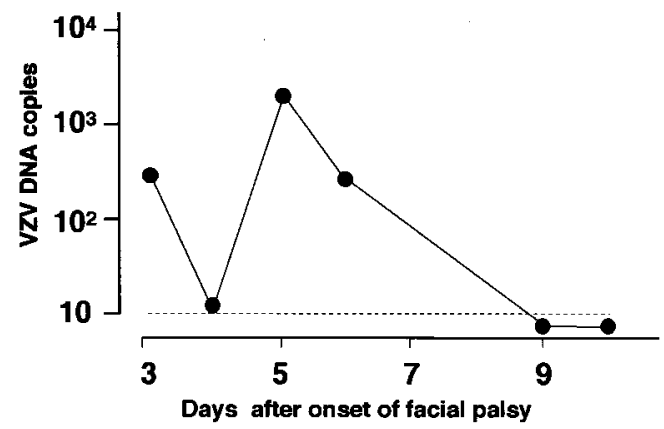

FIG. 1. Transition of VZV DNA load in patients with RHS and ZSH. @, VZV DNA copies, expressed as the copy number/50 $\mu 1$ of saliva (logarithmic scales). Dotted lines indicate the minimum detection level of VZV DNA. (A) Patient with RHS in whom zoster and facial palsy appeared simultaneously (day 0). This patient was treated with acyclovir by infusion at $750 \mathrm{mg}$ per day for 7 days. The VZV DNA levels gradually decreased and became undetectable on day 8. (B) Patient with RHS who exhibited zoster 6 days after the onset of facial palsy. Acyclovir treatment (4,000 $\mathrm{mg}$ in tablets daily for 7 days) started on day 5 before the appearance of zoster because VZV DNA was detected in saliva obtained on day 4. The VZV DNA levels peaked 1 day before the appearance of skin lesions. (C) A patient with ZSH treated with acyclovir by infusion at 750 mg per day for 5 days. The VZV copy number gradually decreased and became undetectable on day 6. (D) A patient with ZSH who did not receive antiviral therapy. The VZV copy number peaked on day 5.

of VZV DNA in each patient was compared between the two groups. As shown in Fig. 2, the viral copy number ranged from 38 to $1,365,800$ copies $/ 50 \mu$ l of saliva in RHS and from 10 to 79,650 copies $/ 50 \mu$ l of saliva in ZSH. The difference in viral copy number between patients with RHS and those with ZSH was not significant $(P>0.05$, Mann-Whitney $U$ test).

\section{DISCUSSION}

The facial nerve consists of two roots: the motor division and the nervus intermedius, which contains parasympathetic fibers and sensory fibers. The cell bodies of the sensory fibers are in the geniculate ganglion, and some afferent fibers supply the mucous membranes of the oropharynx and the skin of the external auditory meatus and around the ear. VZV reactivation in the geniculate ganglia and subsequent inflammation of the facial nerve in the temporal bone are suspected to cause facial palsy $(2,8)$, while VZV migrates from the geniculate ganglia into the skin around the ear or into the oropharynx via the sensory fibers, where it replicates and produces zoster in RHS. In the present study, VZV DNA tended to be detectable in saliva from RHS patients who had zoster in the oropharyngeal epithelium, and the saliva from these patients contained a high copy number of the viral DNA. These results demonstrate the reliability of the TaqMan PCR assay because VZV replicates at the oropharyngeal lesions and is shed into the saliva. We have also shown that VZV DNA is detectable in saliva from patients with RHS who have zoster around the ear and from those with $\mathrm{ZSH}$, suggesting that reactivated VZV in the geniculate ganglia may migrate into the oropharyngeal epithelium without producing zoster at the site.

Using the TaqMan PCR assay, the present study shows that the VZV copy number in saliva increases after the onset of facial palsy and peaks near the day of the appearance of zoster in RHS patients who exhibit facial palsy followed by zoster. In patients who exhibit zoster and facial palsy simultaneously, the VZV load gradually decreases after the onset or peaks several days later. Judging from these results, the viral load in saliva from patients with RHS may peak in accordance with the appearance of zoster. These results are consistent with those from a previous study that found a decrease in the amount of VZV DNA after the onset of varicella (10). In patients who exhibit facial palsy several days after the appearance of zoster, viral replication may already have decreased at the onset of the palsy. Therefore, VZV DNA is less frequently detected in such patients. These findings suggest that the VZV load in saliva reflects the kinetics of viral reactivation.

Two patterns of the VZV load were also observed in PCRpositive patients with ZSH: a decrease after the first visit and a peak after it. These results indicate that the correlation between the kinetics of VZV reactivation and the onset of facial palsy in patients with $\mathrm{ZSH}$ is similar to that in patients with RHS. Based on the findings that VZV DNA is less fre- 


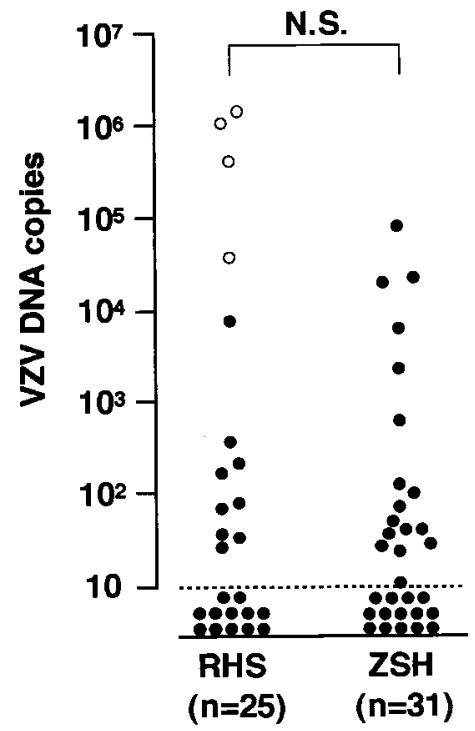

FIG. 2. Quantitation of VZV DNA in saliva samples from patients with RHS and ZSH. The highest copy number in each patient was plotted (logarithmic scales). Samples below the dotted line were negative for VZV DNA. Open circles indicate patients who had zoster in the oropharyngeal epithelium. N.S., not significant.

quently detected in RHS patients who exhibit facial palsy several days after the appearance of zoster, the VZV load may have decreased at the onset in some PCR-negative patients with $\mathrm{ZSH}$.

We also found that the amounts of VZV DNA in saliva were not significantly different between RHS and ZSH. The data suggest that VZV reactivation in ZSH does occur at levels similar to those in RHS and that the VZV load does not play a major role in causing the different manifestations of RHS and ZSH. It has been reported that a VZV-specific T-cell response is correlated with the risk of herpes zoster (7). In addition, depression of cellular immunity against VZV has been demonstrated in RHS and ZSH (9). Therefore, different cellular immunoreactions to VZV at the mucocutaneous site may correlate with the appearance of zoster in facial palsy patients with VZV reactivation. Further studies on cellular immunity to VZV are needed to confirm this hypothesis.

Our results suggest the clinical utility of the TaqMan PCR assay for analysis of VZV replication during antiviral therapy. Acyclovir therapy has resulted in marked suppression of vire- mia in varicella (10). In the present study, acyclovir reduced the VZV copy number in most cases of RHS and ZSH. These findings suggest that the TaqMan PCR assay may be useful in assessing the efficacy of acyclovir therapy.

In conclusion, the present study shows that the VZV load in saliva reflects the kinetics of viral reactivation in patients with RHS and ZSH. In addition, our findings suggest that the VZV load is not the major cause of differences between RHS and $\mathrm{ZSH}$.

\section{ACKNOWLEDGMENTS}

We thank Erina Katoh, Keiko Miyazawa, and Shigeru Yoshida for their excellent technical assistance.

This work was supported by a Grant-in-Aid for Scientific Research, Ministry of Education, Science, Sports and Culture, and by The Akiyama Foundation.

\section{REFERENCES}

1. Davison, A. J., and J. E. Scott. 1986. The complete DNA sequence of varicella-zoster virus. J. Gen. Virol. 67:1759-1816.

2. Furuta, Y., T. Takasu, K. C. Sato, S. Fukuda, Y. Inuyama, R. Hondo, and K. Nagashima. 1992. Detection of varicella-zoster virus DNA in human geniculate ganglia by polymerase chain reaction. J. Infect. Dis. 166:1157-1159.

3. Furuta, Y., S. Fukuda, S. Suzuki, T. Takasu, Y. Inuyama, and K. Nagashima. 1997. Detection of varicella-zoster virus DNA in patients with acute peripheral facial palsy by the polymerase chain reaction, and its use for early diagnosis of zoster sine herpete. J. Med. Virol. 52:316-319.

4. Furuta, Y., F. Ohtani, H. Kawabata, S. Fukuda, and T. Bergström. 2000. High prevalence of varicella-zoster virus reactivation in herpes simplex virusseronegative patients with acute peripheral facial palsy. Clin. Infect. Dis. 30:529-533.

5. Furuta, Y., F. Ohtani, Y. Mesuda, S. Fukuda, and Y. Inuyama. 2000. Early diagnosis of zoster sine herpete and antiviral therapy for the treatment of facial palsy. Neurology 55:708-710.

6. Hawrami, K., and J. Breuer. 1999. Development of a fluorogenic polymerase chain reaction assay (TaqMan) for the detection and quantitation of varicella zoster virus. J. Virol. Methods 79:33-40.

7. Hayward, A. R., and M. Herberger. 1987. Lymphocyte responses to varicella zoster virus in the elderly. J. Immunol. 7:174-178.

8. Hunt, J. R. 1907. On herpetic inflammations of the geniculate ganglion: a new syndrome and its complication. J. Nerv. Ment. Dis. 34:73-96.

9. Ikeda, M., K. Hiroshige, Y. Abiko, and K. Onoda. 1996. Impaired specific cellular immunity to the varicella-zoster virus in patients with herpes zoster oticus. J. Laryngol. Otol. 110:918-921.

10. Kimura, H., S. Kido, T. Ozaki, N. Tanaka, Y. Ito, R. K. Williams, and T. Morishima. 2000. Comparison of quantitations of viral load in varicella and zoster. J. Clin. Microbiol. 38:2447-2449.

11. Morgan, M., and D. Nathwani. 1992. Facial palsy and infection: the unfolding story. Clin. Infect. Dis. 14:263-271.

12. Murakami, S., N. Honda, M. Mizobuchi, Y. Nakashiro, N. Hato, and K. Gyo. 1998. Rapid diagnosis of varicella zoster virus infection in acute facial palsy. Neurology 51:1202-1205.

13. Terada, K., T. Niizuma, S. Kawano, N. Kataoka, T. Akisada, and Y. Orita. 1998. Detection of varicella-zoster virus DNA in peripheral mononuclear cells from patients with Ramsay Hunt syndrome or zoster sine herpete. J. Med. Virol. 56:359-363. 